Zwar scheinen Profifußballer unter den Sportlern noch am ehesten ein erhöhtes Arthroserisiko zu tragen, aber gesichert ist dies nicht.

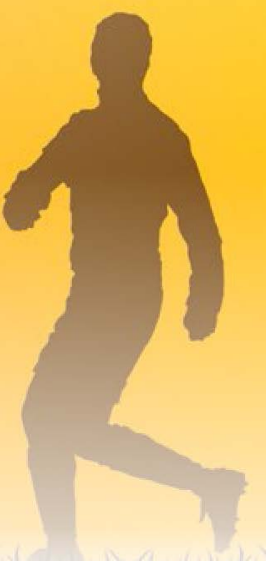

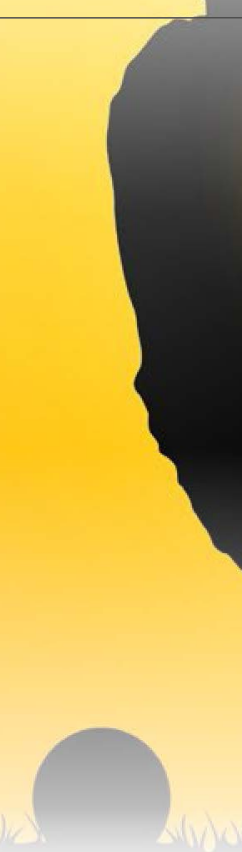

\section{Arthrose durch Sport: Wer ist gefährdet?}

\section{Bergen bestimmte Sportarten ein erhöhtes Arthroserisiko? Um möglichen Zusammenhängen auf die Spur zu kommen, haben britische Forscher eine Metaanalyse zu 32 Sportarten durchgeführt - allerdings mit wenig befriedi- genden Ergebnissen.}

$\mathrm{D}$ as Team um Gui Tran von der Universität Leeds hat sich alle Mühe gegeben, um seine Analyse zum Arthroserisiko durch Sport auf solide Beine zu stellen. Eingangs wurden sieben Kriterien definiert, die für eine hohe Qualität der beteiligten Studien bürgen sollten: So wurden beispielsweise nur prospektive Studien berücksichtigt, die mit einer Vergleichsgruppe arbeiteten und mehr als 100 Teilnehmer umfassten. Dennoch bleibt das Ergebnis der Metaanalyse schwammig, wobei sich zumindest einige Tendenzen feststellen lassen.

Nur so viel lässt sich aufgrund der vorhandenen Evidenz sagen: Das Risiko, an einer Arthrose zu erkranken scheint im Spitzensport erhöht. In erster Linie gefährdet sind Fußballer, weniger Leichtathleten. Für den Freizeitsport bleibt der Zusammenhang nach wie vor unklar. Hier fanden sich lediglich Hinweise darauf, dass frühere Sportverletzungen das Arthroserisiko erhöhen können. Auch hier waren es in erster Linie die Fußballer, die nach Kreuzbandverletzungen und Meniskektomien eine gewisse Risikoerhöhung zeigten.

Fußball ist in diesem Kontext die am besten untersuchte Sportart: 15 von 46 Studien (Profi- und Freizeitsport zusammengenommen) widmeten sich dem
Zusammenhang mit einer Arthrose, in zwölf Studien fand sich eine positive Assoziation. Mit einer gepoolten Prävalenz von 0,35 war die Arthrose hier am häufigsten von allen Sportarten anzutreffen. Die relative Risikoerhöhung lag bei signifikanten $42 \%$.

Zwölf Kohorten schlossen Langstreckenläufer ein. Dabei ergab sich nur in zwei Untersuchungen eine Assoziation zur Hüft- und Kniearthrose, die restlichen zehn Studien fanden keinerlei Zusammenhang. Bei den Leichtathleten legten drei von vier Studien ein erhöhtes Risiko für die Arthrose in Ellenbogenund Hüftgelenken nah. In Studien mit Lang- und Mittelstreckenläufern bestand auf Spitzenniveau kein erhöhtes Arthroserisiko (RR 0,86; p = 0,56).

Für andere Sportarten war die Evidenz deutlich geringer. Für Tennis fand sich keine Assoziation, ebenso wenig für Gewichtheben, Schwimmen oder Schießen; dabei konnten jeweils nur zwei Studien ausgewertet werden. Zehn Studien hatten sich explizit mit der Intensität der sportlichen Aktivität auseinandergesetzt. Davon berichteten neun eine positive Assoziation zum Arthroserisiko. In zwei Studien waren die Zahl der gelaufenen Kilometer und das Tempo mit der Inzidenz Arthrosen in Hüfte und Knie verknüpft, allerdings nur bei Spitzensportlern, nicht im Freizeitsport.

Fünf Untersuchungen schließlich beschäftigten sich mit früheren Sportverletzungen. Eine Studie fand ein um $64 \%$ erhöhtes Arthroserisiko bei Fußballern nach einer Meniskektomie, eine andere bezifferte die Gonarthrosegefahr nach einer Kreuzbandverletzung auf rund 50 $\%$. Gemäß einer multivariaten Analyse galt dies jedoch nicht für Fußballspieler, deren Kreuzbandverletzung operativ versorgt wurde.

Fazit: Von 46 Studien zu den verschiedensten Sportarten belegen 31 ein erhöhtes Arthroserisiko. Die Risikoerhöhung betrug in der Metaanalyse für alle Sportarten zusammengenommen $37 \%$. Während beim Fußball ein Zusammenhang mit der Gelenkerkrankung zu bestehen scheint, konnte dieser beispielsweise für den Laufsport nicht nachgewiesen werden. Die Qualität der Evidenz, so die Forscher, sei jedoch durchweg gering bis sehr gering, dies müsse man bei der Interpretation der Ergebnisse berücksichtigen. Was fehlt, sind qualitativ höherwertige, vor allem prospektive Studien. Dem möglichen Schaden durch sportliche Aktivität, das betonen die Autoren, sei in jedem Fall deren substanzieller Nutzen für Körper und Geist entgegenzustellen.

Dr. Elke Oberhofer

Tran G et al. Does sports participation (including level of performance and previous injury) increase risk of osteoarthritis? A systematic review and meta-analysis. Br J Sports Med 2016; doi: 10.1136/bjsports-2016-096142 